\title{
Metastatic Cervical and Supraclavicular Lymphadenopathy from Prostate Mimicking Lymphoma: A Case Series
}

\author{
Abubakar Sadiq Muhammad1*, Ngwobia Peter Agwu1', Abdullahi Abdulwahab-Ahmed1, \\ Khalid Abdullahi', Kabiru Abdullahi' ${ }^{2}$, Ahmed Mohammed Umar' ${ }^{1}$, Ismaila Arzika Mungadi ${ }^{1}$ \\ ${ }^{1}$ Urology Unit, Departments of Surgery, Usmanu Danfodiyo University Teaching Hospital, Sokoto, Nigeria \\ ${ }^{2}$ Urology Unit, Departments of Histopathology, Usmanu Danfodiyo University Teaching Hospital, Sokoto, Nigeria \\ Email: *asmgusau@gmail.com
}

How to cite this paper: Muhammad, A.S., Agwu, N.P., Abdulwahab-Ahmed, A., Abdullahi, K., Abdullahi, K., Umar, A.M. and Mungadi, I.A. (2019) Metastatic Cervical and Supraclavicular Lymphadenopathy from Prostate Mimicking Lymphoma: A Case Series. Open Journal of Urology, 9, 93-101. https://doi.org/10.4236/oju.2019.96011

Received: February 1, 2019

Accepted: June 27, 2019

Published: June 30, 2019

Copyright $\odot 2019$ by author(s) and Scientific Research Publishing Inc. This work is licensed under the Creative Commons Attribution International License (CC BY 4.0).

http://creativecommons.org/licenses/by/4.0/

\section{Open Access}

\begin{abstract}
Prostate cancer is the second most common cancer in men worldwide and the commonest cause of mortality in men. It is the commonest diagnosed cancer in African men. The earliest and common sites of metastasis are the axial skeleton and regional lymph nodes. Initial presentation of the metastatic disease with cervical or supraclavicular lymphadenopathy is rarely reported. We report 3 cases of metastatic cancer of the prostate presenting initially to physicians with huge cervical and supraclavicular lymph nodes enlargement which on biopsy revealed metastatic adenocarcinoma. On further evaluation, there were non-bothersome storage symptoms, elevated prostate specific antigen and abnormal digital rectal examination. Transrectal ultrasound (TRUS)-guided biopsy of hypoechoic nodules revealed high-grade adenocarcinoma of the prostate on histopathological examination. The cervical and supraclavicular lymphadenopathy resolved after commencement of androgen deprivation therapy. We advocate for prostate cancer screening in African men above 50 years of age presenting with cervical or supraclavicular lymphadenopthy to primary care physicians even in the absence of lower urinary tract symptoms.
\end{abstract}

\section{Keywords}

Metastasis, Cervical Lymphadenopathy, Supraclavicular Lymphadenopathy, Cancer of the Prostate, Lymphoma Mimicker

\section{Introduction}

Prostate cancer is the second most common cancer in men and the most com- 
mon cause of mortality in men above 50 years of age in the Western world [1]. Prostate cancer is the most commonly diagnosed male cancer in Nigeria with 100,000 new cases reported each year [2] [3]. Prostate cancer is known to be aggressive in blacks and most patients present with metastatic disease at presentation [4] [5] [6]. The initial metastasis is to the axial skeleton and pelvic lymph nodes [7]. Initial presentation with cervical or supraclavicular lymphadenopathy is rarely reported [8] [9] [10]. There are less than 50 cases reported in literature [11]. Patients will initially present to general practitioners, physicians, general surgeons, maxillofacial surgeons, Ear, Nose and Throat surgeons before proper evaluation and subsequent referral to urologist [10]. This is at the expense of progression of the disease because of delay in accurate diagnosis. High index of suspicion for prostate cancer is necessary for men above 50 years presenting with cervical or supraclavicular lymph node enlargement even in the absence of urinary symptoms [9] [10].

We present three cases of prostate carcinoma in our center (Usmanu Danfodiyo University Teaching Hospital, Sokoto, northwest Nigeria: UDUTH) initially presenting as metastatic disease to lymph nodes in the head-neck region.

\section{Case Series}

\subsection{Case 1}

This is a 70-year-old farmer who presented to physician first with huge cervical and supraclavicular lymphadenopathy which was suspected lymphoma. He was referred by physicians to maxillofacial surgeons who proceeded to do incisional biopsy of the lymph nodes which revealed metastatic adenocarcinoma. There was history of mild non-bothersome storage symptoms, low back pain, left leg swelling and weight loss. Prostate Specific Antigen (PSA) was done at the maxillofacial unit which revealed value of $31 \mathrm{ng} / \mathrm{ml}$. This necessitated his referral to Urologists.

On examination, there was huge matted, hard, right cervical and supraclavicular lymphadenopathy mass measuring $20 \times 20 \mathrm{~cm}$. This is shown in Figure 1 below. Digital rectal examination revealed an enlarged, hard and nodular prostate. He had eight-core protocol TRUS of the prostate which revealed distorted echotexture, multiple hypoechoic nodules in the peripheral zones bilaterally, breached capsule and infiltration of the right seminal vesicle. The histopathological examination of the prostate specimen revealed adenocarcinoma of the prostate, Gleason sum of 9 as shown in Figure 2(a) and Figure 2(b). Diagnosis of metastatic prostate cancer with left cervical and supraclavicular lymphadenopathy was done. He had bilateral total orchidectomy (BTO) after appropriate counseling; verbal and written consent. The lymph node mass regressed partially and resolved fully after one and two months respectively as shown in Figure 3. The patient represented after a year with difficulty in walking, bone pains, and generalized body weakness. The repeat PSA after BTO was $42 \mathrm{ng} / \mathrm{ml}$ and serum testosterone was $0.408 \mathrm{ng} / \mathrm{ml}$. The diagnosis of Castration Resistant Prostate 


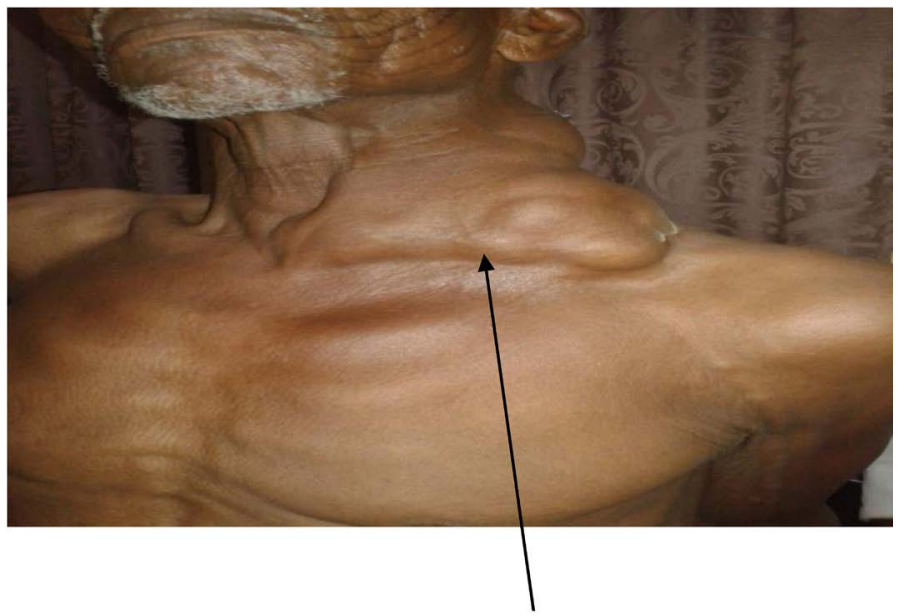

Cervical and supraclavicular lymph node mass

Figure 1. Preoperative clinical photograph of case 1, showing huge matted lymph node mass from left cervical and supraclavicular lymphadenopathy.

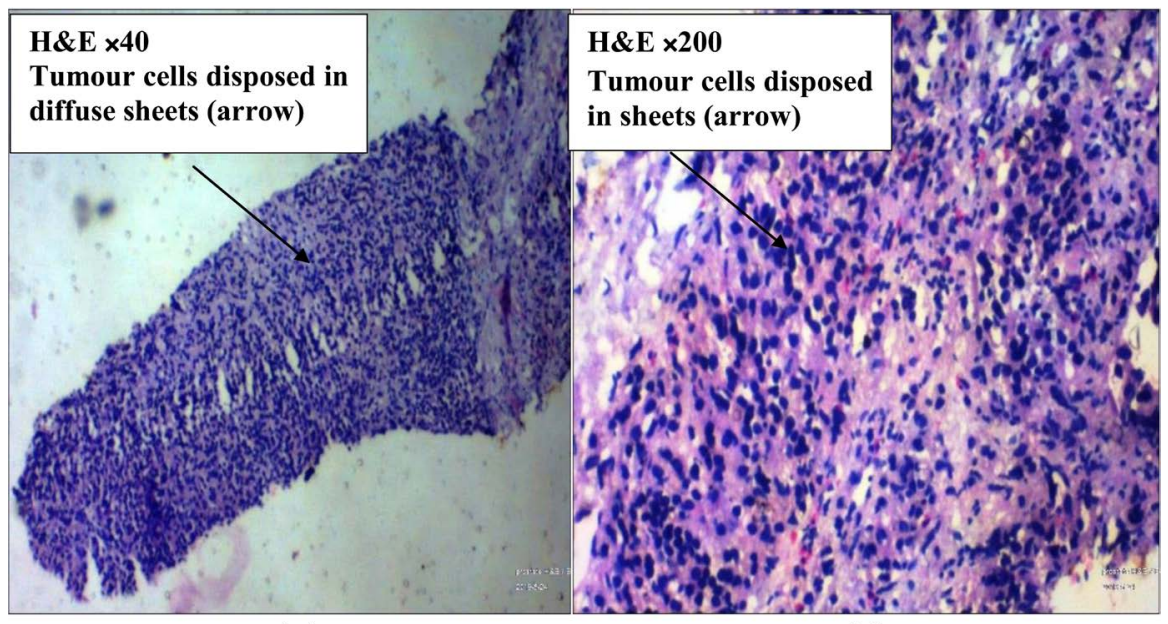

(a)

(b)

Figure 2. (a) Photomicrograph of prostate biopsy specimen (low magnification); (b) Photomicrograph of the same patient specimen (high magnification).

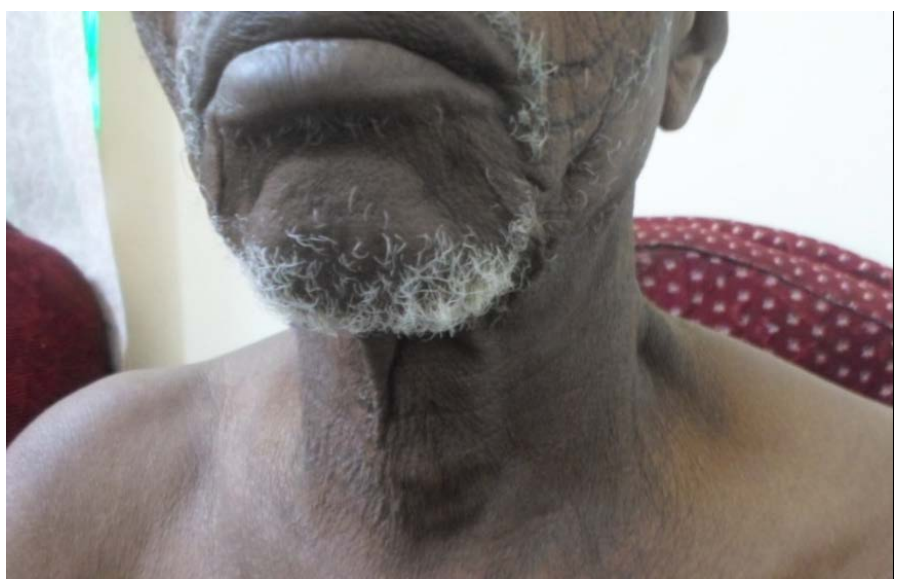

Figure 3. Case 1 post bilateral total orchidectomy showing complete resolution of the left cervical and supraclavicular lymph node mass. 
Cancer (CRPC) was made. Symptoms resolved following addition of bicalutamide $50 \mathrm{mg}$ daily to have Maximal Androgen Blockade (MAB). The patient was also placed on alendronate $70 \mathrm{mg}$ weekly and dihydrocodeine (DF118) $30 \mathrm{mg}$ twice a day for pain. He is presently on the bicalutamide daily.

\subsection{Case 2}

This is a 70-year-old man who was referred by physicians to general surgeons and subsequently to urology unit with history of cough, weight loss, terminal painless haematuria, left neck swelling and lower urinary tract symptoms. There was history of painless childhood haematuria. The patient was cachectic, pale with generalized lymphadenopathy involving cervical, inguinal and para-aortic lymph nodes. The lymph nodes are of varying sizes, discrete, hard, mobile, the biggest measuring $2.5 \mathrm{~cm} \times 3 \mathrm{~cm}$. The cervical lymph node is shown in Figure 4 below. Digital Rectal Examination and TRUS were suspicious. Prostate Specific Antigen done was $40 \mathrm{ng} / \mathrm{ml}$. The abdominal ultrasound revealed multiple mesenteric and paraaortic lymph nodes, normal bladder and apparently normal prostate of $22 \mathrm{~g}$. Excisional biopsy of the cervical lymph node was done by the general surgeons which revealed metastatic adenocarcinoma. The lymph node specimen is shown in Figure 5 below. He had eight-core protocol TRUS-guided

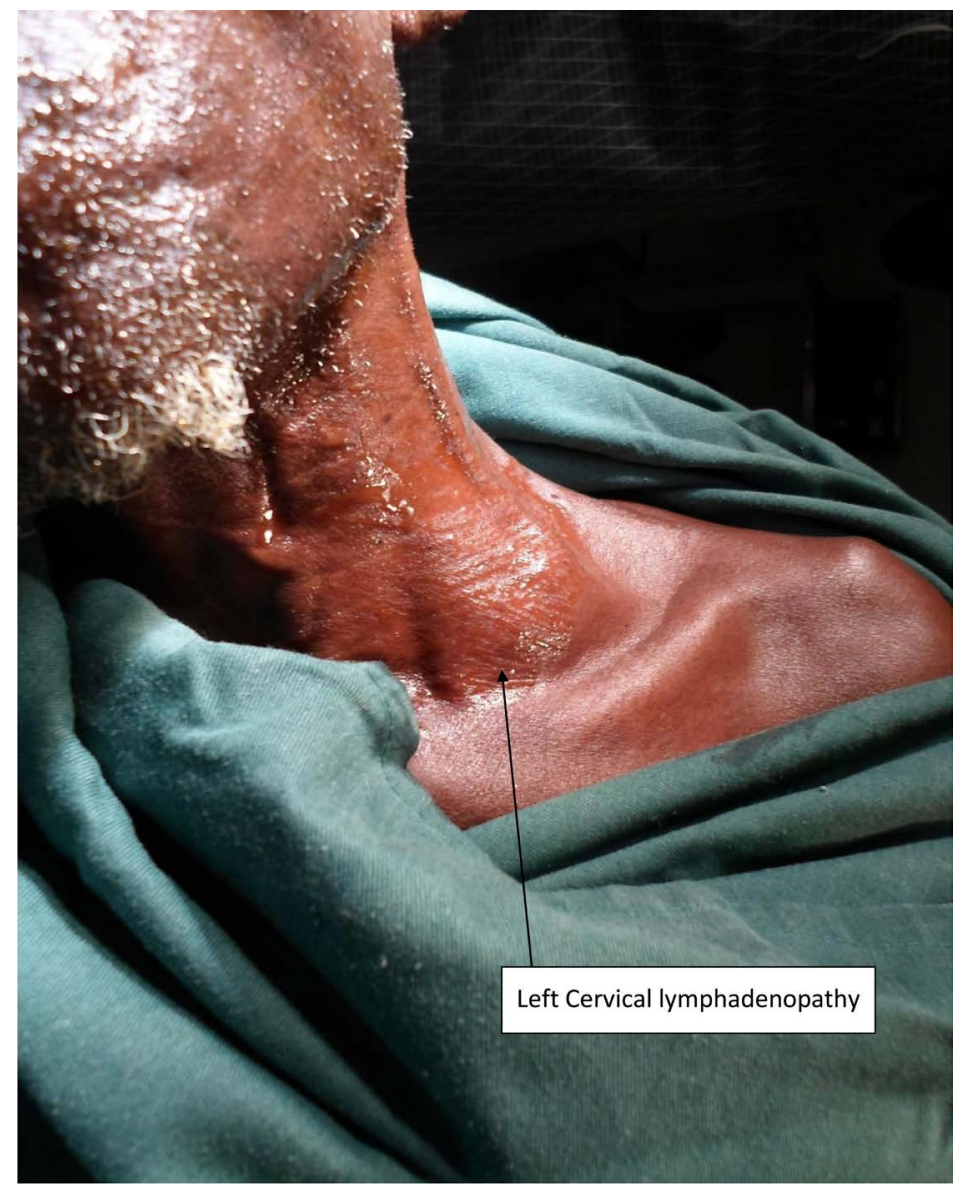

Figure 4. Preoperative clinical photograph of case 2 showing left cervical lymphadenopathy. 
prostate biopsy and the histology revealed adenocarcinoma as shown in Figure 6(a) and Figure 6(b). Sputum microscopy revealed Streptococcus pneumonia and was negative for acid-fast bacilli. Gene expert and chest $\times$ ray were not suggestive of Koch's disease. He had BTO after counseling and informed consent. He had a resolution of symptoms and PSA nadir was $21.4 \mathrm{ng} / \mathrm{ml} 1$-month postoperatively. The PSA rose to $42.59 \mathrm{ng} 4$ months post operatively and the patient was subsequently lost to follow up during evaluation for CRPC.

\subsection{Case 3}

This is a 55-year-old man who presented to physicians with 1-month history of chest pain, neck swelling, neck pain and parasthesia in upper and lower limbs. There was history of storage symptoms, low back pain, lower limb weakness, difficulty in walking, weight loss and anorexia. He was in painful distress with limitation of range of motion in the neck. There was flexion deformity of the neck and the patient walked with support. There was huge, hard, matted left cervical and supraclavicular lymphadenopathy $10 \times 8 \times 8 \mathrm{~cm}$ as shown in Figure 7

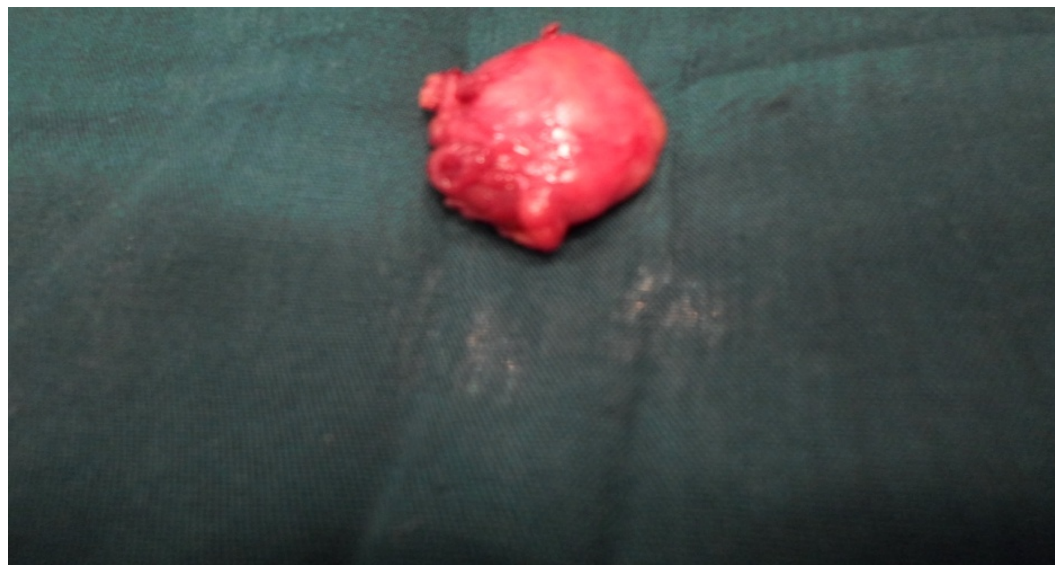

Figure 5. Excisional biopsy lymph node specimen of case 2.

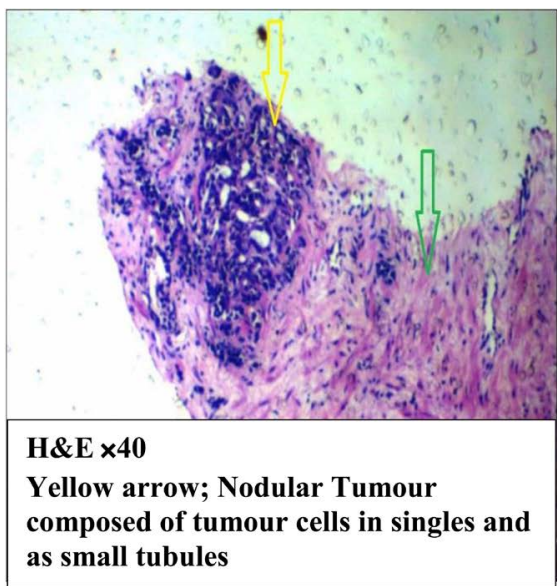

(a)

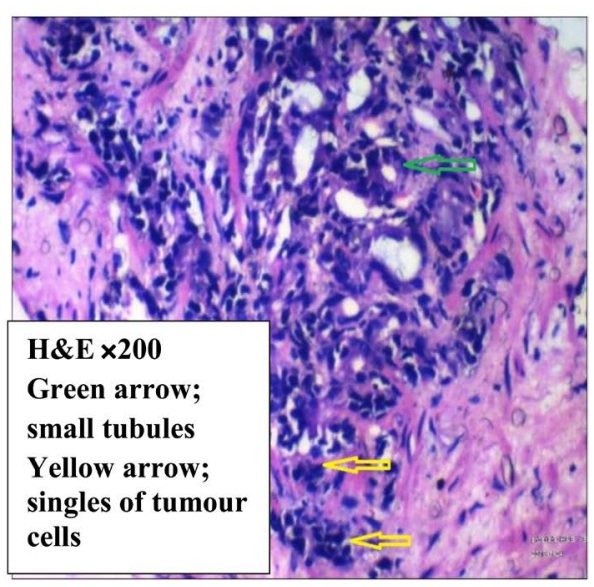

(b)

Figure 6. (a) Photomicrograph of prostate biopsy specimen (low magnification); (b) Photomicrograph of the same patient specimen (high magnification). 
below. There was also hard and matted left inguinal lyphadenopathy measuring $6 \times 4 \mathrm{~cm}$. Digital rectal examination revealed lax anal sphinter, enlarged, hard and craggy prostate. The chest $\mathrm{x}$-ray and abdominal ultrasound revealed absence of chest or abdominal metastasis. Cervical and lumbo-sacral x-rays showed osteosclerotic changes. The PSA done was $113.28 \mathrm{ng} / \mathrm{ml}$. He had eight-core TRUS-guided prostate biopsy which revealed adenocarcinoma, Gleason score of 7 as shown in Figure 8(a) and Figure 8(b). He was counseled and the patient opted for medical castration with Goserelin acetate (Zoladex) $10.8 \mathrm{mg}$. He received courses of bicalutamide $150 \mathrm{mg}$, Ketoconazole $400 \mathrm{mg}$, dexaamethasone 8 mg for 48 hours for impending cord compression before the histological confirmation of the disease. He had initial resolution of the symptoms and lymph nodes (Figure 9) which were more remarkable a month after the medical castration.

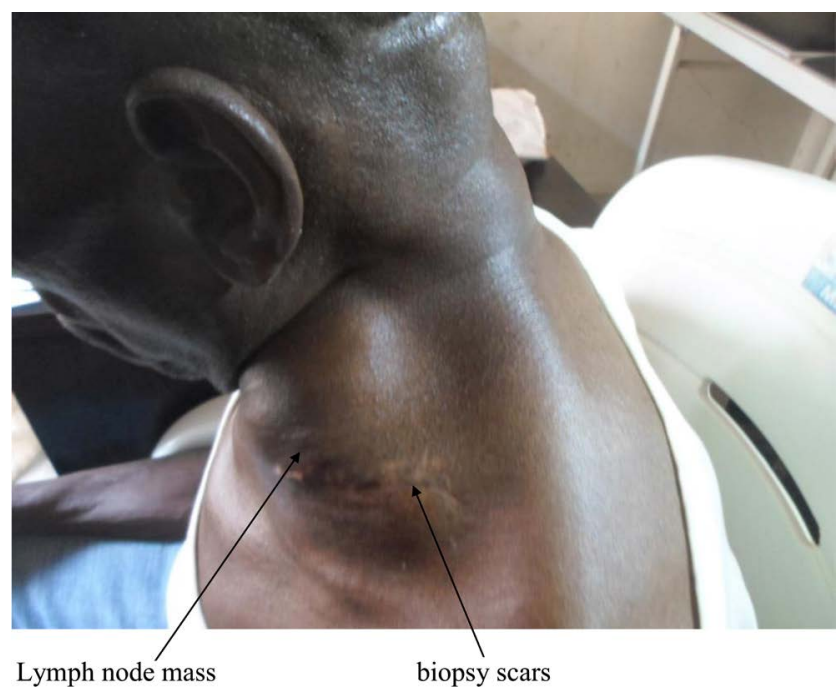

Figure 7. Clinical photograph of case 3 showing a matted left supraclavicular lymphadenopathy and incisional biopsy scar at presentation.

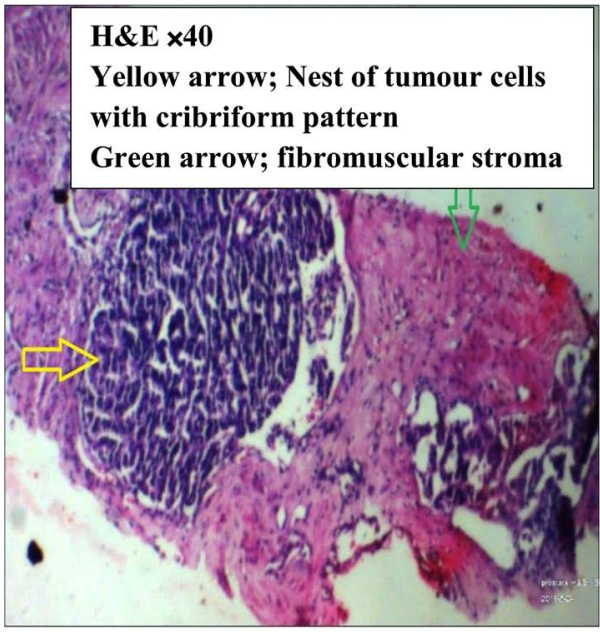

(a)

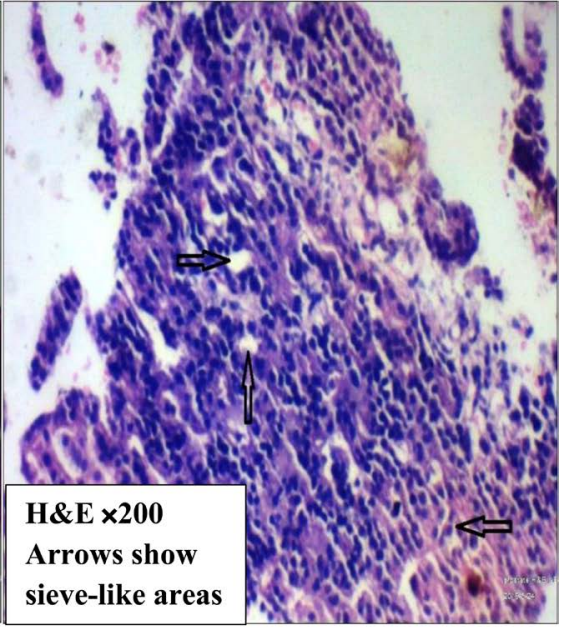

(b)

Figure 8. (a) Photomicrograph of prostate biopsy specimen (low magnification); (b) Photomicrograph of the same patient specimen (high magnification). 


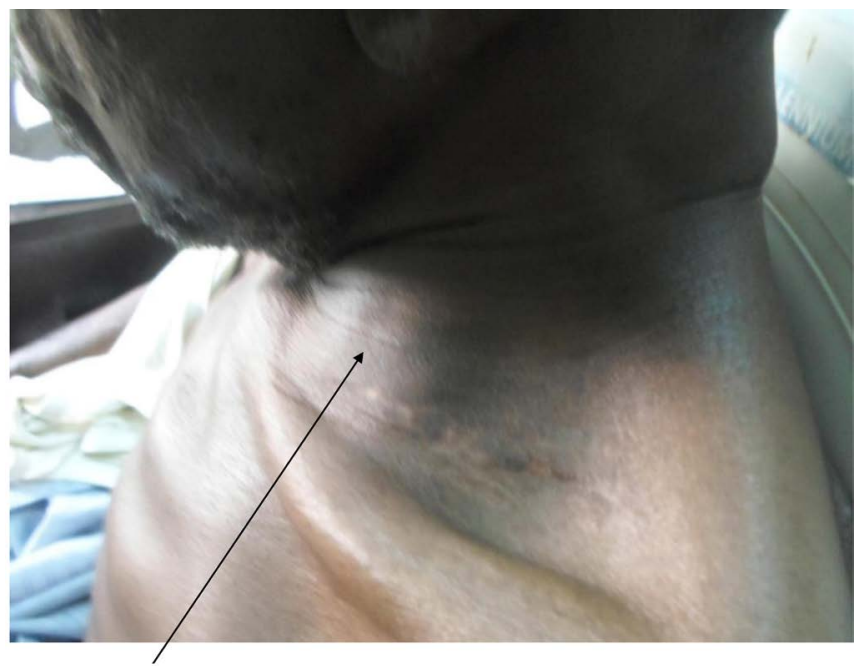

Residual lymph node mass

Figure 9. Clinical photograph of case 3 after first dose of medical castration with Goserelin acetate, showing residual left supraclavicular lymphadenopathy.

The PSA was $2.73 \mathrm{ng} / \mathrm{ml}$ and $0.533 \mathrm{ng} / \mathrm{ml}$ after the first and second dose of the Zoladex. The patient was co-managed with Neurosurgical team on account of residual neck pain and limitation of neck the movement.

\section{Discussion}

Prostate cancer is known to be aggressive in blacks and patients present with advanced disease [5] [6] [12]. Screening for prostate cancer is not a common practice and there is no guideline or protocol for it in Nigeria and most African countries [3]. Prostate cancer is known to metastasize early to lower spine and regional lymph nodes [11]. Metastasis to the cervical spine, supraclavicular lymph nodes and other non-regional lymph nodes is rarely reported [7]. It was reported that prostate cancer involved cervical lymph nodes in $0.4 \%$ of patients and the patients usually have widespread metastatic disease [10].

In this study, the patients are above 50 years, with two of them up to 70 years which is in keeping with what was reported in the literature except for a case that was reported in a 42-year-old patient [5] [11] [12] [13]. The patients had mild non-bothersome lower urinary tract symptoms (LUTS) which leads to initial presentations to physicians and other surgeons, suspicion of lymphoma and disseminated tuberculosis. This was noticed in the previous studies, that prostate cancer patients with cervical or supraclavicular lymphadenopathy may not present with LUTS which leads to suspicion of lymphoma or metastasis from aero-digestive tract [10]. Most of the cases reported were confirmed by immunohistochemistry of the metastatic cervical or supraclavicular nodal biopsy specimen which was positive for PSA [7] [10] [11] [12] [13] [14]. This test is not routinely done in my institution as such, the diagnosis was confirmed after TRUS- guided prostate biopsy which revealed adenocarcinoma on histology.

The symptoms of the patient resolved after androgen deprivation therapy as 
reported by previous studies [10]. On the contrary, one of the patients has cervical spine involvement and still having some symptom of parasthesia in the upper limbs and limitation of range of motion in the neck. This patient may have more comprehensive treatment with BTO and Neurosurgical co-management. $\mathrm{He}$ is currently being co-managed with the Neuro-surgeons and undergoing counseling for BTO. The longest follow up recorded in this study was 30 months which is within the range of average survival of 19.8 - 29.7 years reported in a previous study [11]. One of our patients was lost to follow up but two are still coming for follow up. Left cervical and supraclavicular lymphadenothy were the commonest nodes involved as observed in the present study [10] [12]. The involvement of the left neck lymph nodes was by retrograde spread due to the proximity of these nodes to the left subclavian vein as it was joined by the thoracic duct [14].

From the aforementioned cases, there should be high index of suspicion for prostate cancer in men above 50 years of age that present with cervical and or supraclavicular lymph nodes enlargement even in the absence of lower urinary tract symptoms. Therefore, we advocate screening for prostate cancer in African men above the age of 50 years presenting with cervical or supraclavicular lymphadenopathy even in the absence of LUTS [9]. The screening involved PSA, digital rectal examination and TRUS-guided prostate biopsy.

\section{Conclusion}

Cervical or supraclavicular lymphadenopathy may be the initial indication of metastatic prostate cancer. Therefore, the initial presentation may be to physicians or other surgeons. Prostate cancer should be suspected in men above 50 years of age with neck swelling even in the absence of lower urinary symptoms and other symptoms of the malignancy. Prompt referral to urology, confirmation of the malignancy and androgen deprivation therapy is necessary for resolution of symptoms which is dramatic.

\section{Conflicts of Interest}

The authors declare no conflicts of interest regarding the publication of this paper.

\section{References}

[1] Siegel, R.L., Miller, K.D., Khan, R. and Jemal, A. (2018) Cancer Statistics. $C A: A$ Cancer Journal for Clinicians, 68, 7-30. https://doi.org/10.1891/9780826121646.0002

[2] Awodele, O., Adeyomoye, A.A., Awodele, D.F., Fayankinnu, V.B. and Dolapo, D.C. (2011) Cancer Distribution Pattern in South-Western Nigeria. Tanzania Journal of Health Research, 13, 125-131. https://doi.org/10.4314/thrb.v13i2.55226

[3] Ikuerowo, S.O., Omisanjo, O.A., Bioku, M.J., Ajala, M.O., Nonyelim Mordi, V.P. and Esho, J.O. (2013) Prevalence and Characteristics of Prostate Cancer among Participants of a Community-Based Screening in Nigeria Using Serum Prostate Specific 
Antigen and Digital Rectal Examination. The Pan African Medical Journal, 15, 129. https://doi.org/10.11604/pamj.2013.15.129.2489

[4] Oranusi, C.K., Ugezu, A.I. and Nwofor, A. (2012) Diagnosis of Prostate Cancer with Needle Biopsy: Should All Cases Be Biopsied before Treatment. Nigerian Journal of Clinical Practice, 15, 48-50. https://doi.org/10.4103/1119-3077.94097

[5] Stephen, A.J. and Klein, E.A. (2016) Epedemiology, Aetiology and Prevention of Prostate Cancer. In: Wein, A.J., Partin, A.W., Peters, C.A., Eds., Campbell-Walsh Urology, 11th Edition, Elsevier Sunders, Philadelphia, 7270-7310.

[6] Badmus, T.A., Adesunkanmi, A.R.K., Yusuf, B.M., Oseni, G.O., Eziyi, A.K., Bakare, T.I.B., et al. (2010) Burden of Prostate Cancer in Southwestern Nigeria. Urology, 76, 412-416. https://doi.org/10.1016/j.urology.2010.03.020

[7] Lad, M., Sharma, A. and Patten, D.K. (2014) A Surprising Diagnosis: Metastatic Prostate Cancer Causing Cervical Lymphadenopathy. BMJ Case Reports, 2014, bcr2013201630.

[8] Carleton, J., van der Riet, P. and Dahm, P. (2005) Metastatic Prostate Cancer Presenting as an Asymptomatic Neck Mass. Prostate Cancer and Prostatic Diseases, 8 , 293-295. https://doi.org/10.1038/sj.pcan.4500805

[9] Joshi, P. and Lele, V. (2012) Prostatic Adenocarcinoma Masquerading as Generalized lymphadenopathy and Mimicking lymphoma on FDG PET/CT: Diagnosis, Staging, and Evaluation of Therapy Response by FDG PET/CT. Nephro-Urology Monthly, 4, 482-484. https://doi.org/10.5812/numonthly.2303

[10] Abusultan, M., Hanzel, P., Durcansky, D. and Hajtman, A. (2017) Left Supraclavicular Lymphadenopathy as the Only Clinical Presentation of Prostate Cancer: A Case Report. Acta Medica Martiniana, 17, 41-44. https://doi.org/10.1515/acm-2017-0011

[11] Sepúlveda, 1., Gorgal, T., Pires, V. and Rodrigues, F. (2015) Cancer Metastatic to the Cervical Lymph Nodes. Case Reports in Urology, 2015, Article ID: 263978. https://doi.org/10.1155/2015/263978

[12] Jones, H. and Anthony, P.P. (1992) Metastatic Prostatic Carcinoma Presenting as Left-Sided Cervical Lymphadenopathy: A Series of 11 Cases. Histopathology, 21, 149-154. https://doi.org/10.1111/j.1365-2559.1992.tb00363.x

[13] Lin, Y.Y., Lin, D.S., Kang, B.H. and Lin, Y.S. (2011) Neck Mass as the First Presentation of Metastatic Prostatic Adenocarcinoma. Journal of the Chinese Medical Association, 74, 570-573. https://doi.org/10.1016/j.jcma.2011.09.012

[14] Dubhashi, S.P., Kumar, H. and Nath, S.R. (2012) Prostate Cancer Presenting as Cervical Lymphadenopathy. American Journal of Case Reports, 13, 206-208. https://doi.org/10.12659/AJCR.883334 